\title{
Multiplicity of positive solutions for a nonlinear differential equation with nonlinear boundary conditions
}

by D. R. Dunninger and Haiyan Wang (East Lansing, Mich.)

\begin{abstract}
We study the existence and multiplicity of positive solutions of the nonlinear equation $u^{\prime \prime}(x)+\lambda h(x) f(u(x))=0$ subject to nonlinear boundary conditions. The method of upper and lower solutions and degree theory arguments are used.

1. Introduction. In this paper we are concerned with the existence and multiplicities of positive solutions of the nonlinear boundary value problem

$$
\left\{\begin{array}{l}
u^{\prime \prime}(x)+\lambda h(x) f(u(x))=0, \quad 0<x<1, \\
B_{0} u \equiv \alpha u(0)-\beta u^{\prime}(0)=\lambda g_{1}(u(0)), \\
B_{1} u \equiv \gamma u(1)+\delta u^{\prime}(1)=\lambda g_{2}(u(1)) .
\end{array}\right.
$$

By a positive solution of (1) we understand a solution $u \in C^{2}[0,1]$ with $u>0$ in $(0,1)$. As a consequence of the maximum principle, our hypotheses below will imply that every nonnegative solution of (1) is positive.

The main results follow.

Theorem 1.1. Assume

$\left(\mathrm{A}_{1}\right) \quad \alpha, \beta, \gamma, \delta \geq 0$ and $\varrho \equiv \gamma \beta+\alpha \gamma+\alpha \delta>0$.

$\left(\mathrm{A}_{2}\right) \quad \lambda$ is a positive parameter.

$\left(\mathrm{A}_{3}\right) \quad h:[0,1] \rightarrow[0, \infty)$ is continuous and does not vanish identically on any subset (of positive measure) of $[0,1]$.

$\left(\mathrm{A}_{4}\right) \quad f:[0, \infty) \rightarrow(0, \infty)$ is continuous.

$\left(\mathrm{A}_{5}\right) \quad g_{1}, g_{2}:[0, \infty) \rightarrow(0, \infty)$ are continuous.

$\left(\mathrm{A}_{6}\right) \quad f_{\infty}=\lim _{u \rightarrow \infty} f(u) / u=\infty$.
\end{abstract}

Then there exists a positive number $\lambda^{*}$ such that (1) has at least two positive solutions for $0<\lambda<\lambda^{*}$, at least one positive solution for $\lambda=\lambda^{*}$, and no positive solutions for $\lambda>\lambda^{*}$.

1991 Mathematics Subject Classification: 34A34, 34B15.

Key words and phrases: nonlinear boundary value problems, multiplicity of positive solutions, upper and lower solutions, degree theory. 
Theorem 1.2. Suppose $f \equiv 0$. Assume $\left(\mathrm{A}_{1}\right),\left(\mathrm{A}_{2}\right),\left(\mathrm{A}_{5}\right)$ and $\left(\mathrm{A}_{7}\right) \quad\left(g_{1}\right)_{\infty}=\lim _{u \rightarrow \infty} g_{1}(u) / u=\infty,\left(g_{2}\right)_{\infty}=\lim _{u \rightarrow \infty} g_{2}(u) / u=\infty$.

Then the conclusions of Theorem 1.1 are valid.

Note that we do not require any monotonicity conditions on $f, g_{1}, g_{2}$. Theorem 1.1 was proved for a variety of nonlinear differential equations under homogeneous Dirichlet boundary conditions in $[2,4,7,8]$ and for a system of differential equations under general homogeneous boundary conditions in [5]. Related results for (1) can be found in [1,3] and the references therein.

Our purpose here is to study the influence of the nonlinearities in the boundary condition. As a direct consequence of Theorems 1.1 and 1.2, we can easily obtain corresponding results for positive radial solutions of the following elliptic system on an annulus:

$$
\begin{cases}\Delta u+\lambda h(|x|) f(u)=0 & \text { in } 0<R_{1}<|x|<R_{2}, \\ \alpha u+\beta \frac{\partial u}{\partial n}=\lambda g_{1}(u) & \text { on }|x|=R_{1}, \\ \gamma u+\delta \frac{\partial u}{\partial n}=\lambda g_{2}(u) & \text { on }|x|=R_{2},\end{cases}
$$

where $\partial / \partial n$ denotes the outer normal derivative on the boundary.

Indeed, the search for positive radial solutions $u=u(r)$ of (2) leads to

$$
\left\{\begin{array}{l}
u^{\prime \prime}(r)+\frac{n-1}{r} u^{\prime}(r)+\lambda h(r) f(u(r))=0, \quad R_{1}<r<R_{2} \\
\alpha u\left(R_{1}\right)-\beta u^{\prime}\left(R_{1}\right)=\lambda g_{1}\left(u\left(R_{1}\right)\right), \quad \gamma u\left(R_{2}\right)+\delta u^{\prime}\left(R_{2}\right)=\lambda g_{2}\left(u\left(R_{2}\right)\right) .
\end{array}\right.
$$

By applying the change of variables $s=-\int_{r}^{R_{2}}\left(1 / t^{n-1}\right) d t$, followed by the change of variables $t=(m-s) / m$, where $m=-\int_{R_{1}}^{R_{2}}\left(1 / t^{n-1}\right) d t$, system (3) can be brought into the form

$$
\left\{\begin{array}{l}
u^{\prime \prime}(x)+\lambda h^{*}(x) f(u(x))=0, \quad 0<x<1, \\
\alpha u(0)-\beta \frac{R_{1}^{1-n}}{m} u^{\prime}(0)=\lambda g_{1}(u(0)), \quad \gamma u(1)+\delta \frac{R_{2}^{1-n}}{m} u^{\prime}(1)=\lambda g_{2}(u(1)),
\end{array}\right.
$$

where $h^{*}(t)=m^{2} r^{2(n-1)}(m(1-t)) h(r(m(1-t)))$. It is easy to check that $h^{*}$ satisfies $\left(\mathrm{A}_{3}\right)$, and that the corresponding condition $\left(\mathrm{A}_{1}\right)$ is met.

In proving our results, we employ upper and lower solution methods together with degree theory arguments. We establish Theorem 1.1 first. Preliminary results are given in Section 2. In Section 3, an existence and nonexistence result is proved. Upper and lower solutions are discussed in Section 4. The proof of Theorem 1.1 is given in Section 5. Also in Section 5 we indicate the modifications needed in order to prove Theorem 1.2. 
2. Preliminaries. We first note that (1) is equivalent to the integral equation

(4) $u(x)=\lambda(P(u(0), u(1)) x+Q(u(0), u(1)))+\lambda \int_{0}^{1} k(x, y) h(y) f(u(y)) d y$

where

$$
P(s, t)=\frac{\alpha g_{2}(t)-\gamma g_{1}(s)}{\varrho}, \quad Q(s, t)=\frac{(\gamma+\delta) g_{1}(s)+\beta g_{2}(t)}{\varrho}
$$

and $k(x, y)$ is the Green's function

$$
k(x, y)=\frac{1}{\varrho} \begin{cases}(\gamma+\delta-\gamma x)(\beta+\alpha y), & y \leq x \\ (\beta+\alpha x)(\gamma+\delta-\gamma y), & x \leq y .\end{cases}
$$

Consequently, (4) is equivalent to the fixed point equation

$$
T u=u
$$

in the usual Banach space $X=C([0,1])$ with $\|u\|=\sup _{x \in[0,1]}|u(x)|$, where $T: X \rightarrow X$ is given by

(7) $T u(x)=\lambda(P(u(0), u(1)) x+Q(u(0), u(1)))+\lambda \int_{0}^{1} k(x, y) h(y) f(u(y)) d y$.

We need the following elementary lemmas:

Lemma 2.1. Suppose $k(x, y)$ is the Green's function defined in (6). Then

$$
\begin{array}{ll}
k(x, y)>0 & \text { for all } x, y \in[1 / 4,3 / 4], \\
k(x, y) \geq \frac{1}{16} k(z, y) & \text { for all } x \in[1 / 4,3 / 4], y, z \in[0,1] .
\end{array}
$$

Proof. Clearly,

$$
k(x, y) \geq \frac{1}{\varrho}\left(\delta+\frac{\gamma}{4}\right)\left(\beta+\frac{\alpha}{4}\right)>0 \quad \text { for all } x, y \in[1 / 4,3 / 4] .
$$

Moreover, for $x \in[1 / 4,3 / 4]$, we have

$$
k(x, y) \geq \frac{1}{\varrho} \begin{cases}(\delta+\gamma / 4)(\beta+\alpha y), & 0 \leq y \leq 1 / 4, \\ (\delta+\gamma / 4)(\beta+\alpha / 4), & 1 / 4<y<3 / 4, \\ (\beta+\alpha / 4)(\gamma+\delta-\gamma y), & 3 / 4 \leq y \leq 1 .\end{cases}
$$

On the other hand, for any $z, y \in[0,1]$, we have

$$
k(z, y) \leq \frac{1}{\varrho}(\beta+\alpha y)(\gamma+\delta-\gamma y)
$$

from which (8) easily follows.

Lemma 2.2. Consider $P(s, t) x+Q(s, t)$, where $P, Q$ are defined in (5). Then

$$
P(s, t) x+Q(s, t) \geq \min (Q(s, t), P(s, t)+Q(s, t)) \geq 0,
$$




$$
P(s, t) x+Q(s, t) \leq \max (Q(s, t), P(s, t)+Q(s, t))
$$

for all $x$ in $[0,1]$ and $s, t \geq 0$. Further,

$$
P(s, t) x+Q(s, t) \geq \frac{1}{4}(P(s, t) z+Q(s, t))
$$

for all $x \in[1 / 4,3 / 4], z \in[0,1], s, t \geq 0$.

Proof. The estimates (9)-(11) follow easily from the linearity (in $x$ ) of $P(s, t) x+Q(s, t)$ for each fixed $s, t \geq 0$, and the nonnegativity of $g_{1}$ and $g_{2}$.

\section{Existence and nonexistence}

Theorem 3.1. Assume $\left(\mathrm{A}_{1}\right)-\left(\mathrm{A}_{6}\right)$. Then for $\lambda$ sufficiently small, (1) has at least one positive solution, whereas for $\lambda$ sufficiently large, (1) has no positive solutions.

We shall need some lemmas to prove this result. It is obvious that the operator $T$ defined in (7) is completely continuous. Let $K$ be the cone defined by

$$
K=\left\{u \in X: u \geq 0, \min _{x \in[1 / 4,3 / 4]} u(x) \geq \frac{1}{16}\|u\|\right\}
$$

and let $C$ be the cone defined by

$$
C=\{u \in X: u \geq 0\} .
$$

LEMma 3.2. $T(C) \subset K$.

Pr o of. Choose $u \in C$. Utilizing (8), (9) and (11), for $x \in[1 / 4,3 / 4]$ we have

$$
\begin{aligned}
T u(x) & \geq \lambda\left(\frac{1}{4}\left(P(u(0), u(1)) z+Q(u(0), u(1))+\frac{1}{16} \int_{0}^{1} k(z, y) h(y) f(u(y)) d y\right)\right. \\
& \geq \frac{1}{16} T u(z)
\end{aligned}
$$

for all $z \in[0,1]$, and so

$$
\min _{x \in[1 / 4,3 / 4]} T u(x) \geq \frac{1}{16}\|T u\|,
$$

i.e., $T(u) \in K$; hence $T(C) \subset K$.

The following lemma is well known and crucial in our arguments; see [6] for proofs and further discussions of the fixed point index.

Lemma 3.3. Let $X$ be a Banach space and $K$ a cone in $X$. For $r>0$, define $K_{r}=\{x \in K:\|x\|<r\}$. Assume that $T: \bar{K}_{r} \rightarrow K$ is a compact map such that $T x \neq x$ for $x \in \partial K_{r}$.

(i) If $\|x\| \leq\|T x\|$ for $x \in \partial K_{r}$, then $i\left(T, K_{r}, K\right)=0$.

(ii) If $\|x\| \geq\|T x\|$ for $x \in \partial K_{r}$, then $i\left(T, K_{r}, K\right)=1$. 
Proof of Theorem 3.1. In the following we set

$$
M=\max _{(x, y) \in[0,1] \times[0,1]} k(x, y)>0, \quad m=\min _{(x, y) \in[1 / 4,3 / 4] \times[1 / 4,3 / 4]} k(x, y)>0 .
$$

For $q>0$, let

$$
I(q)=M \max _{u \in K,\|u\|=q}\left(\int_{0}^{1} h(y) f(u(y)) d y\right)>0 .
$$

For any number $r_{1}>0$, let

$$
K_{r_{1}}=\left\{u \in K:\|u\|<r_{1}\right\} .
$$

We then choose $\sigma>0$ so that

$$
\sigma \leq \frac{r_{1}}{2 I\left(r_{1}\right)}
$$

and

$$
\sigma \max (Q(u(0), u(1)), P(u(0), u(1))+Q(u(0), u(1))) \leq r_{1} / 2, \quad u \in \partial K_{r_{1}} .
$$

Then for $\lambda \leq \sigma$ and $u \in \partial K_{r_{1}}$, we have, recalling (10),

$$
T u(x) \leq \frac{r_{1}}{2}+\sigma M \int_{0}^{1} h(y) f(u(y)) d y \leq \frac{r_{1}}{2}+\sigma I\left(r_{1}\right) \leq r_{1},
$$

which implies

$$
\|T(u)\| \leq r_{1}=\|u\|
$$

for $u \in \partial K_{r_{1}}$. Thus Lemma 3.3 implies

$$
i\left(T, K_{r_{1}}, K\right)=1 \text {. }
$$

Since $f_{\infty}=\infty$, there is a $p>0$ such that $f(u) \geq \eta u$ for $u \geq p$, where $\eta$ is chosen so that

$$
\frac{\lambda m \eta}{16} \int_{1 / 4}^{3 / 4} h(y) d y \geq 1
$$

Choose $r_{2} \geq 16 p$ and set $K_{r_{2}}=\left\{u \in K:\|u\|<r_{2}\right\}$. If $u \in \partial K_{r_{2}}$, then

$$
\min _{x \in[1 / 4,3 / 4]} u(x) \geq \frac{1}{16}\|u\| \geq p
$$

and for any $x \in[1 / 4,3 / 4]$, we have

$$
\begin{aligned}
T u(x) & \geq \lambda m \int_{1 / 4}^{3 / 4} h(y) f(u(y)) d y \\
& \geq \lambda m \eta \int_{1 / 4}^{3 / 4} h(y) u(y) d y \geq \frac{\lambda m \eta}{16}\|u\| \int_{1 / 4}^{3 / 4} h(y) d y \geq\|u\|,
\end{aligned}
$$


which implies $\|T(u)\| \geq\|u\|$ for $u \in \partial K_{r_{2}}$. An application of Lemma 3.3 again shows that

$$
i\left(T, K_{r_{2}}, K\right)=0 .
$$

Since we can adjust $r_{1}$ and $r_{2}$ so that $r_{1}<r_{2}$, it follows from the additivity of the fixed point index that $i\left(T, K_{r_{2}} \backslash \bar{K}_{r_{1}}, K\right)=-1$, and thus $T$ has a fixed point in $K_{r_{2}} \backslash \bar{K}_{r_{1}}$, i.e., (1) has at least one positive solution.

From $\left(\mathrm{A}_{4}\right)$ and $\left(\mathrm{A}_{6}\right)$, there is a constant $c$ such that $f(u) \geq c u$ for $u \geq 0$. Let $u \in X$ be a positive solution of (4). By Lemma 3.2, $u \in K$. Now choose $\lambda$ large enough so that

$$
\frac{\lambda m c}{16} \int_{1 / 4}^{3 / 4} h(y) d y>1
$$

Then for $x \in[1 / 4,3 / 4]$, we have

$$
u(x) \geq \lambda m c \int_{1 / 4}^{3 / 4} h(y) u(y) d y \geq \frac{\lambda m c}{16}\|u\| \int_{1 / 4}^{3 / 4} h(y) d y>\|u\|,
$$

which is an obvious contradiction. Hence (1) has no positive solutions for $\lambda$ sufficiently large.

4. Upper and lower solutions. We say that the function $\bar{u} \in C^{2}[0,1]$ is an upper solution of (1) if

$$
\begin{gathered}
\bar{u}^{\prime \prime}(x)+\lambda h(x) f(\bar{u}(x)) \leq 0, \quad 0<x<1, \\
B_{0} \bar{u} \geq \lambda g_{1}(\bar{u}(0)), \quad B_{1} \bar{u} \geq \lambda g_{2}(\bar{u}(1)),
\end{gathered}
$$

and $\underline{u} \in C^{2}[0,1]$ is a lower solution if

$$
\begin{gathered}
\underline{u}^{\prime \prime}(x)+\lambda h(x) f(\underline{u}(x)) \geq 0, \quad 0<x<1, \\
B_{0} \underline{u} \leq \lambda g_{1}(\underline{u}(0)), \quad B_{1} \underline{u} \leq \lambda g_{2}(\underline{u}(1)) .
\end{gathered}
$$

We now establish several lemmas that will be used in the sequel. Let $\bar{u}, \underline{u}$ be upper and lower solutions for (1) and define

$$
\begin{aligned}
& f^{*}(u(x))= \begin{cases}f(\bar{u}(x)), & u(x) \geq \bar{u}(x), \\
f(u(x)), & \underline{u}(x) \leq u(x) \leq \bar{u}(x), \\
f(\underline{u}(x)), & u(x) \leq \underline{u}(x),\end{cases} \\
& g_{1}^{*}(u(x))= \begin{cases}g_{1}(\bar{u}(x)), & u(x) \geq \bar{u}(x), \\
g_{1}(u(x)), & \underline{u}(x) \leq u(x) \leq \bar{u}(x), \\
g_{1}(\underline{u}(x)), & u(x) \leq \underline{u}(x),\end{cases} \\
& g_{2}^{*}(u(x))= \begin{cases}g_{2}(\bar{u}(x)), & u(x) \geq \bar{u}(x), \\
g_{2}(u(x)), & \underline{u}(x) \leq u(x) \leq \bar{u}(x), \\
g_{2}(\underline{u}(x)), & u(x) \leq \underline{u}(x) .\end{cases}
\end{aligned}
$$


Consider the following problem:

$$
\left\{\begin{array}{l}
u^{\prime \prime}(x)+\lambda h(x) f^{*}(u(x))=0, \quad 0<x<1, \\
B_{0} u=\lambda g_{1}^{*}(u(0)), \\
B_{1} u=\lambda g_{2}^{*}(u(1)) .
\end{array}\right.
$$

LEMMA 4.1. If there is a solution $u$ of $(13)$, then $\underline{u}(x) \leq u(x) \leq \bar{u}(x)$ for $x \in[0,1]$. In other words, $u$ is a solution of (1).

Proof. We first prove that $u(x) \leq \bar{u}(x)$. Suppose to the contrary that $u\left(x_{0}\right)>\bar{u}\left(x_{0}\right)$ for some $x_{0} \in[0,1]$. Then there are four cases:

(i) $u(x)>\bar{u}(x)$ for all $x \in[0,1]$. In this case, we have

$$
f^{*}(u(x))=f(\bar{u}(x)), \quad g_{1}^{*}(u(0))=g_{1}(\bar{u}(0)), \quad g_{2}^{*}(u(0))=g_{2}(\bar{u}(0)) .
$$

Therefore

$$
(\bar{u}-u)^{\prime \prime} \leq 0, \quad B_{0}(\bar{u}-u) \geq 0, \quad B_{1}(\bar{u}-u) \geq 0,
$$

which, by the maximum principle, implies the contradiction $\bar{u}(x) \geq u(x)$ for all $x \in[0,1]$. Then

(ii) $u(x)>\bar{u}(x)$ for all $x \in(a, 1]$, where $0<a<1$, and $u(a)=\bar{u}(a)$.

$$
\begin{gathered}
(\bar{u}-u)^{\prime \prime} \leq 0 \quad \text { for all } x \in[a, 1] \\
\bar{u}(a)-u(a)=0, \quad B_{2}(\bar{u}-u) \geq 0
\end{gathered}
$$

which again implies the contradiction $\bar{u}(x) \geq u(x)$ for all $x \in[a, 1]$.

In a similar way we handle the other two cases:

(iii) $u(x)>\bar{u}(x)$ for all $x \in[0, a)$ where $0<a<1$, and $u(a)=\bar{u}(a)$.

(iv) $u(x)>\bar{u}(x)$ for all $x \in(a, b)$, where $0<a, b<1$, and $u(a)=$ $\bar{u}(a), u(b)=\bar{u}(b)$.

By the same arguments we see that $\underline{u}(x) \leq u(x)$ for $x \in[0,1]$. Since $\underline{u}(x) \leq u(x) \leq \bar{u}(x)$ for $x \in[0,1]$, it follows that $f^{*}=f, g_{1}^{*}=g_{1}, g_{2}^{*}=g_{2}$, and so $u$ is also a solution of (1).

LEMMA 4.2. If there exist upper and lower solutions $\bar{u}$ and $\underline{u}$ of (1) with $\underline{u}(x) \leq \bar{u}(x)$ for $x \in[0,1]$, then there is a solution $u$ to (1) such that

$$
\underline{u}(x) \leq u(x) \leq \bar{u}(x) \quad \text { for all } x \in[0,1] .
$$

Pr o of. Consider problem (13). The equivalent integral equation is

$$
\begin{aligned}
u(x)= & \lambda\left(P^{*}(u(0), u(1)) x+Q^{*}(u(0), u(1))\right) \\
& +\lambda \int_{0}^{1} k(x, y) h(y) f^{*}(u(y)) d y
\end{aligned}
$$

where

$$
P^{*}(s, t)=\frac{\alpha g_{2}^{*}(t)-\gamma g_{1}^{*}(s)}{\varrho}, \quad Q^{*}(s, t)=\frac{(\gamma+\delta) g_{1}^{*}(s)+\beta g_{2}^{*}(t)}{\varrho}
$$


and $k(x, y)$ is the corresponding Green's function. Let

$$
T^{*} u(x)=\lambda\left(P^{*}(u(0), u(1)) x+Q^{*}(u(0), u(1))\right)+\lambda \int_{0}^{1} k(x, y) h(y) f^{*}(u(y)) d y .
$$

Then $T^{*}: C[0,1] \rightarrow C[0,1]$ is completely continuous. Since $f^{*}, g_{1}^{*}, g_{2}^{*}$ are bounded, so is $T^{*}$. By Schauder's fixed point theorem, $T^{*}$ has a fixed point $u$, which is a solution of (13). By Lemma 4.1, $u$ is also a solution of (1).

5. Multiplicity. Since we are seeking positive solutions, we shall make the convention that $f(u)=f(0), g_{1}(u)=g_{1}(0), g_{2}(u)=g_{2}(0)$ if $u<0$.

We first need the following a priori estimate.

LEMmA 5.1. There is a constant $b_{I}>0$ such that $\|u\| \leq b_{I}$ for all solutions $u$ of (1) where $\lambda$ belongs to a compact subset I of $(0, \infty)$.

P r o o f. Suppose there is an unbounded sequence $\left\{u_{n}\right\}$ of solutions of (1), and thus of (4), with the corresponding $\lambda_{n}$ belonging to a compact subset of $(0, \infty)$. By Lemma 3.2, $u_{n} \in K$. Since $f_{\infty}=\infty$, there is a $p>0$ such that $f(u) \geq \eta u$ for $u \geq p$, where $\eta$ satisfies

$$
\frac{\lambda_{n} \eta}{4} \int_{1 / 4}^{3 / 4} k\left(\frac{1}{2}, y\right) h(y) d y \geq 2 \text { for all } n
$$

Since $\left\|u_{n}\right\| \rightarrow \infty$ as $n \rightarrow \infty$, there exists an $N>0$ such that $n \geq N$ implies

$$
\min _{x \in[1 / 4,3 / 4]} u_{n}(x) \geq \frac{1}{4}\left\|u_{n}\right\| \geq p
$$

and thus

$$
\begin{aligned}
u_{n}\left(\frac{1}{2}\right) & \geq \lambda_{n} \int_{1 / 4}^{3 / 4} k\left(\frac{1}{2}, y\right) h(y) f\left(u_{n}(y)\right) d y \\
& \geq \frac{\lambda_{n} \eta}{4}\left\|u_{n}\right\| \int_{1 / 4}^{3 / 4} k\left(\frac{1}{2}, y\right) h(y) d y \geq 2\left\|u_{n}\right\|,
\end{aligned}
$$

which is a contradiction.

Now let $\Gamma$ denote the set of $\lambda>0$ such that a positive solution of (1) exists and let $\lambda^{*}=\sup \Gamma$. By Theorem 3.1, $0<\lambda^{*}<\infty$. We claim that $\lambda^{*} \in \Gamma$. To see this, let $\lambda_{n} \rightarrow \lambda^{*}$ where $\lambda_{n} \in \Gamma$. Since the $\lambda_{n}$ are bounded, Lemma 5.1 implies that the corresponding solutions $u_{n}$ are bounded. By the compactness of the integral operator $T$, it easily follows that $\lambda^{*} \in \Gamma$. 
Let $u^{*}$ be a solution of (1) corresponding to $\lambda^{*}$ and define

$$
\begin{gathered}
\tilde{f}(u(x))= \begin{cases}f\left(u^{*}(x)+\varepsilon\right), & u(x) \geq u^{*}(x)+\varepsilon, \\
f(u(x)), & -\varepsilon \leq u(x) \leq u^{*}(x)+\varepsilon, \\
f(-\varepsilon), & u(x) \leq-\varepsilon,\end{cases} \\
\widetilde{g}_{1}(u(x))= \begin{cases}g_{1}\left(u^{*}(x)+\varepsilon\right), & u(x) \geq u^{*}(x)+\varepsilon, \\
g_{1}(u(x)), & -\varepsilon \leq u(x) \leq u^{*}(x)+\varepsilon, \\
g_{1}(-\varepsilon), & u(x) \leq-\varepsilon,\end{cases} \\
\widetilde{g}_{2}(u(x))= \begin{cases}g_{2}\left(u^{*}(x)+\varepsilon\right), & u(x) \geq u^{*}(x)+\varepsilon, \\
g_{2}(u(x)), & -\varepsilon \leq u(x) \leq u^{*}(x)+\varepsilon, \\
g_{2}(-\varepsilon), & u(x) \leq-\varepsilon .\end{cases}
\end{gathered}
$$

Let

$$
\widetilde{T}_{\lambda}(u(x))=\lambda(\widetilde{P}(u(0), u(1)) x+\widetilde{Q}(u(0), u(1)))+\lambda \int_{0}^{1} k(x, y) h(y) \widetilde{f}(u(y)) d y
$$

where

$$
\widetilde{P}(s, t)=\frac{\alpha \widetilde{g}_{2}(t)-\gamma \widetilde{g}_{1}(s)}{\varrho}, \quad \widetilde{Q}(s, t)=\frac{(\gamma+\delta) \widetilde{g}_{1}(s)+\beta \widetilde{g}_{2}(t)}{\varrho} .
$$

Consider

$$
\Omega=\left\{u \in X:-\varepsilon<u(x)<u^{*}(x)+\varepsilon\right\} .
$$

LEMma 5.2. There is an $\varepsilon>0$, sufficiently small, such that if $u \in C[0,1]$ satisfies $\widetilde{T}_{\lambda} u=u$ for some $0<\lambda<\lambda^{*}$, then $u \in \bar{\Omega}$.

Proof. It is clear that $u \geq 0$. To prove that $u \leq u^{*}+\varepsilon$, we first show that $u^{*}+\varepsilon$ is an upper solution of (1). Since $u^{*} \geq 0$, there is a constant $c>0$ so that $f\left(u^{*}(x)\right)>c$ for all $x \in[0,1]$. By uniform continuity, there is an $\varepsilon_{0}$ such that

$$
\left|f\left(u^{*}(x)+\varepsilon\right)-f\left(u^{*}(x)\right)\right|<c\left(\lambda^{*}-\lambda\right) / \lambda
$$

for all $x \in[0,1], 0 \leq \varepsilon \leq \varepsilon_{0}$. Then

$$
\begin{aligned}
\left(u^{*}+\varepsilon\right)^{\prime \prime} & =\left(u^{*}\right)^{\prime \prime}=-\lambda^{*} h f\left(u^{*}\right) \\
& =-\lambda h f\left(u^{*}+\varepsilon\right)+\lambda\left[h f\left(u^{*}+\varepsilon\right)-h f\left(u^{*}\right)\right]+\left(\lambda-\lambda^{*}\right) h f\left(u^{*}\right) \\
& <-\lambda h f\left(u^{*}+\varepsilon\right)+\operatorname{ch}\left(\lambda^{*}-\lambda\right)+\operatorname{ch}\left(\lambda-\lambda^{*}\right)=-\lambda h f\left(u^{*}+\varepsilon\right) .
\end{aligned}
$$

Thus

$$
\left(u^{*}+\varepsilon\right)^{\prime \prime}+\lambda h f\left(u^{*}+\varepsilon\right) \leq 0 .
$$

By the same arguments, we can show that

$$
B_{0}\left(u^{*}+\varepsilon\right) \geq \lambda g_{1}\left(u^{*}(0)+\varepsilon\right), \quad B_{1}\left(u^{*}+\varepsilon\right) \geq \lambda g_{2}\left(u^{*}(1)+\varepsilon\right)
$$

for $\varepsilon>0$ and sufficiently small. Therefore $u^{*}+\varepsilon$ is an upper solution of (1). It follows from Lemma 4.1 that $u \leq u^{*}+\varepsilon$. 
Proof of Theorem 1.1. Let $0<\lambda<\lambda^{*}$. Since $u^{*}$ is an upper solution and 0 is a lower solution, Lemma 4.2 implies the existence of a solution $u_{\lambda}$ of (1) such that $0 \leq u_{\lambda} \leq u^{*}$. Thus for $0<\lambda \leq \lambda^{*}$ a positive solution exists, whereas for $\lambda>\lambda^{*}$ a positive solution does not exist. Moreover, $u_{\lambda} \in \Omega$. We next establish the existence of a second positive solution of (1).

Since $\widetilde{T}_{\lambda}$ is bounded for $\lambda$ in compact intervals,

$$
\operatorname{deg}\left(I-\widetilde{T}_{\lambda}, B\left(u_{\lambda}, R\right), 0\right)=1
$$

for $R$ large enough, where $B\left(u_{\lambda}, R\right)$ is the ball centered at $u_{\lambda}$ with radius $R$ in $C[0,1]$. If there exists a $u \in \partial \Omega$ such that $u=\widetilde{T}_{\lambda}(u)$, then $f=\widetilde{f}, g_{1}=\widetilde{g}_{1}$, $g_{2}=\widetilde{g}_{2}$, and so $u$ is a second positive solution. Now suppose $u \neq \widetilde{T}_{\lambda}(u)$ for all $u \in \partial \Omega$. Then $\operatorname{deg}\left(I-\widetilde{T}_{\lambda}, \Omega, 0\right)$ is well defined. Since Lemma 5.2 implies $\widetilde{T}_{\lambda}$ has no fixed point in $\left.B\left(u_{\lambda}, R\right)\right) \backslash \Omega$, by the excision property of degree we have

$$
\operatorname{deg}\left(I-T_{\lambda}, \Omega, 0\right)=\operatorname{deg}\left(I-\widetilde{T}_{\lambda}, \Omega, 0\right)=1 .
$$

On the other hand, by Lemma 5.1, all solutions of (1) are bounded for $\lambda$ in compact sets, and thus

$$
\operatorname{deg}\left(I-T_{\lambda}, B(0, M), 0\right)=\text { const }
$$

for $M$ large enough. The latter degree must equal 0 , since for $\lambda>\lambda^{*}$ no solutions exist. Finally, by the excision property,

$$
\operatorname{deg}\left(I-T_{\lambda}, B(0, M) \backslash \Omega, 0\right)=-1
$$

and so a second positive solution of system (1) exists for $0<\lambda<\lambda^{*}$.

Proof of Theorem 1.2. Here we briefly consider (1) with $f \equiv 0$. In this case the equivalent operator equation is $u=T u$, where

$$
T u(x)=\lambda\left(\frac{\alpha g_{2}(u(1))-\gamma g_{1}(u(0))}{\varrho} x+\frac{(\gamma+\delta) g_{1}(u(0))+\beta g_{2}(u(1))}{\varrho}\right) .
$$

If we replace the cone defined in (12) by the cone

$$
K=\{u \in X: u=a x+b \geq 0, a, b \in \mathbb{R}, x \in[0,1]\}
$$

then it is obvious that $T(C) \subset K$ is completely continuous. Moreover, if $u \in K$, we note that

$$
\|u\|=\max (u(0), u(1)) .
$$

Now the proof of Theorem 1.2 proceeds along the same lines as that of Theorem 1.1. More precisely, it suffices to replace the previous estimates on $f$ using condition $\left(\mathrm{A}_{6}\right)$ by the corresponding estimates on $g_{1}$ and $g_{2}$ using $\left(\mathrm{A}_{7}\right)$. 


\section{References}

[1] H. A mann, On the existence of positive solutions of nonlinear elliptic boundary value problems, Indiana Univ. Math. J. 21 (1971), 125-146.

[2] -, On the number of solutions of asymptotically superlinear two point boundary value problems, Arch. Rational Mech. Anal. 55 (1974), 207-213.

[3] D. S. Cohen, Generalized radiation cooling of a convex solid, J. Math. Anal. Appl. 35 (1971), 503-511.

[4] H. Dang, K. Schmidt and R. Shivaji, On the number of solutions of boundary value problems involving the p-Laplacian, Electron. J. Differential Equations 1 (1996), $1-9$.

[5] D. R. Dunninger and H. Wang, Multiplicity of positive solutions for an elliptic system, preprint.

[6] D. Guo and V. Lakshmikantham, Nonlinear Problems in Abstract Cones, Academic Press, Orlando, FL, 1988.

[7] K. S. Ha and Y. Lee, Existence of multiple positive solutions of singular boundary value problems, Nonlinear Anal. 28 (1997), 1429-1438.

[8] S. S. Lin, Positive radial solutions and nonradial bifurcation for semilinear elliptic equations in annular domains, J. Differential Equations 86 (1990), 367-391.

Department of Mathematics

Michigan State University

East Lansing, Michigan 48824

U.S.A.

E-mail:dun@math.msu.edu

wangh@math.msu.edu

Reçu par la Rédaction le 29.8.1997 